\title{
SIMULATING SCHEDULE OPTIMIZATION PROBLEM IN STEELMAKING CONTINUOUS CASTING PROCESS
}

\author{
Yang, Z. ; Qiu, H.-L. ${ }^{* *}, \#$; Luo, X.-W. ${ }^{* * *, *} \&$ Shen, D.* \\ * School of Business Administration, Zhejiang Gongshang University, Hangzhou, China \\ ** Department of Business Administration, Tourism College of Zhejiang, Hangzhou, China \\ ${ }^{* * *}$ Department of Logistics Technology, Zhejiang Technical Institute of Economics, Hangzhou, China \\ E-Mail: extra.terrestrial@163.com, qiuhongliang1127@163.com ( ${ }^{\#}$ Corresponding author) \\ chet168@163.com,shend28877193@126.com
}

\begin{abstract}
This paper establishes the models of the steelmaking continuous casting (SCC) process, and proposed the improved algorithms for this problem. The simulation results of a computerized scheduling system are also given to prove the model. The SCC process scheduling problem is very difficult to get a good performance solution in practice. The scheduling of the SCC process requires that each cast plan is processed on time, and the charges should be processed continuously for the same caster in the same cast, as well as the waiting time of the charges cannot be conflicted mutually in the same converters. We propose a quantum-behaved particle swarm optimization (QPSO) and improved algorithm strategy. The results show that the QPSO is very efficient for solving the SCC production scheduling problem, especially for large scale problem.
\end{abstract}

(Received, processed and accepted by the Chinese Representative Office.)

Key Words: SCC, Scheduling Problem, Quantum-Behaved Particle Swarm Optimization

\section{INTRODUCTION}

Steelmaking Continuous Casting (SCC) production scheduling process is an important operation in iron \& steel companies. In this process, we need to decide the sequence, the beginning time, as well as the molten steel machine and scheduling of the molten steel in the iron \& steel production stages. It is very difficult to get a good performance solution in practice in iron \& steel production [1]. Optimization of the scheduling SCC processes can reduce the energy consumption and improve the profit of the company, so many iron \& steel companies have studied the SCC production process for continuous improvement. For example, according to the report of Baoshan Steel Plant in China, one second of SCC production scheduling time saving could bring approximately 5800 dollars profits [2]. However the scheduling of SCC processes has not been solved very well because of its complexity in terms of combinatorial nature, complex constraints, the material continuity and strict practicability requirements, as well as the flow time constrains.

There are many related literature study the optimization for the scheduling of the SCC problems, and many models have been proposed and discussed. However, it is still not very satisfactory when solving the large-scale SCC problems in actual iron \& steel companies. The most commonly used methods for solving the SCC scheduling problems are and mixed-integer linear optimization (MILP) [3-5], artificial intelligence, linear optimization (LP), heuristics $[6,7]$ and simulation methods. The literatures [8-10] illustrate the detailed reviews on these methods.

The two-stage heuristic method is commonly used to solve the machine assignments according to the load equalization and secondly optimized the charges' beginning time. Recently, Li et al. [11] propose a unit-specific event for SSC production scheduling process, and Lin et al. [12] propose a continuous optimal approach for the medium size SSC problem. Then Janak et al. $[13,14]$ extend the approach to large scale industrial batch plant. Comparing 
with the models not use the rolling horizon approach, the final results of the models using the extended rolling horizon approach imply that this improved method can result in the shorter total computing time and better performances of the scheduling solutions. Chen et al. [15] improve the Lagrange Relaxation (LR) approach for the manufacturing job shop scheduling problems, and Fisher [16] employs this method to solve the integer programming production problems. Recently Tang et al. [17] establish a mathematical program model embedded within the LR for SSC production scheduling problem. Almost at the same time, the subgradient method and the bundle method are proposed for job shop scheduling problems [18, 19].

From the observation, however, all the algorithms mentioned above cannot solve the actual SCC process scheduling problems. Form all the previous methods, even employing highly complex algorithm and modern super computers, the compute time used to solve the problems of integer programming are multiplying with increase of the problem size, so the optimization of the SCC process scheduling problems are needed according to the actual steelmaking situations, which is the primary motivation of this research.

\section{PROBLEM DESCRIPTION}

Generally speaking, it includes smelting process (LD), blowing argon (AR), ladle treatment $(\mathrm{LF})$, vacuum $(\mathrm{RH})$, rotary table process (the $\mathrm{CW}$ ) and continuous casting $(\mathrm{CC})$ in the process of steelmaking-continuous casting. The process routes may be different for different steels. The model in this paper is based on the practical production situation of an iron \& steel company in Ma On Shan in China. There are four sets of parallel machines in smelting process, 3 sets of parallel machines in continuous casting process, and two parallel machines for each other process. The parallel machines are suitable for all of the same process. According to the actual production situation, we consider four different process routes: LD $\mathrm{AR}-\mathrm{CW}-\mathrm{CC}, \mathrm{LD}-\mathrm{LF}-\mathrm{CW}-\mathrm{CC}, \mathrm{LD}-\mathrm{LF}-\mathrm{RH}-\mathrm{CW}-\mathrm{CC}, \mathrm{LD}-\mathrm{RH}-\mathrm{CW}-\mathrm{CC}$. Fig. 1 illustrates the schematic of the steelmaking continuous casting process routes.

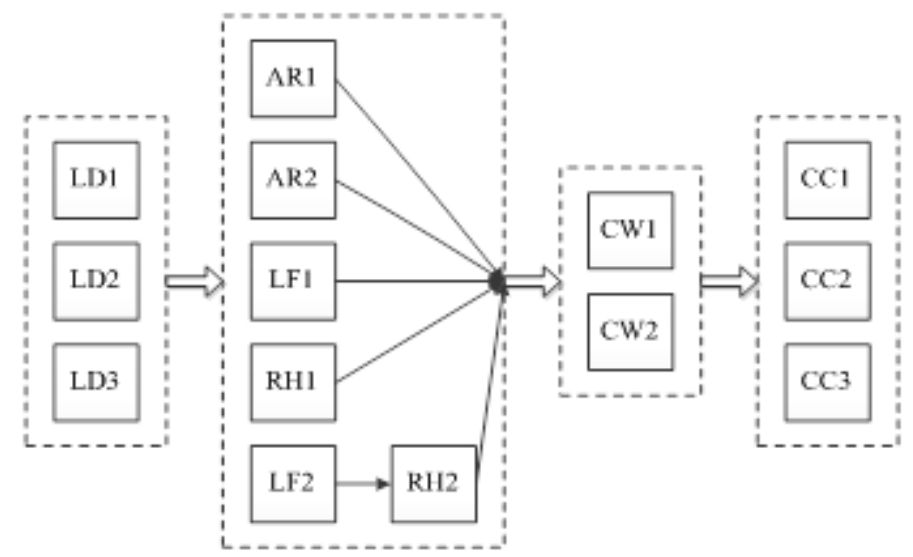

Figure 1: The schematic of the steelmaking continuous casting process routes.

The main purpose of SCC production scheduling is the coordination between smelting, refining, continuous casting production balance problems in order to meet the requirements of the continuous casting molten steel composition and temperature of molten steel, as well as to ensure reasonable cohesion and matching with subsequent rolling process. SCC production scheduling is considered according to the given production plan in this paper, so in this paper, the assumptions are proposed as follows: sequence of charges in casts, sequence of casts and the corresponding continuous casting machine are already known; the beginning time of each cast is known; the assignments of the smelting, refining stage equipment for the castes are not restricted. 


\section{MATHEMATICAL MODEL}

All the symbols used in the model are shown in Table I.

Table I: The symbols of model.

\begin{tabular}{|l|l|}
\hline Symbols & Explanation of symbols \\
\hline$N$ & Total number of the charges \\
\hline$n$ & Number of the charge, index $n=1,2, \ldots, N$ \\
\hline$M$ & Total number of the casts \\
\hline$m$ & Number of the cast, index $m=1,2, \ldots, M$ \\
\hline$k_{f}$ & Total number of processes of the $m$ cast \\
\hline$M_{n}$ & All the casts of the $n$ charge \\
\hline$i, j, k$ & The process of the steel \\
\hline$Q_{m i}$ & The $i$ process of the $m$ cast \\
\hline$r_{m i}$ & The machine number of the $i$ process of the $m$ cast \\
\hline$s_{m i}$ & The beginning time of the $i$ process of the $m$ cast \\
\hline$t_{m i}$ & The finished time of the $i$ process of the $m$ cast \\
\hline$p_{m i}$ & The process time of the $i$ process of the $m$ cast \\
\hline$d_{m i}$ & The difference between the $s_{m i}$ and $t_{m(i-1)}$ \\
\hline
\end{tabular}

The mathematical formulations of model are shown as:

$$
\begin{gathered}
\min Z=\max _{m=1, \cdots, M}\left\{t_{m k_{m}}\right\} \\
\text { s.t. } \quad t_{m k_{m}}=s_{(m+1) k_{m+1}}, \quad m, m+1=1,2, \cdots, M_{n} \\
t_{m i}=s_{m i}+p_{m i}, \quad m=1,2, \cdots, M, \quad 0<i<k_{m} \\
t_{m i}-s_{m(i+1)}=0, \quad m=1,2, \cdots, M, \quad 0<i<k_{m} \\
s_{m i}-t_{m(i+1)} \leq p_{c w}, \quad p_{c w}=p_{5}, m=1,2, \cdots, M \\
s_{m 5}-t_{m 5} \leq 0, \quad p=1,2, \cdots, v_{t}, m=1,2, \cdots, M \\
\left(s_{m_{0} i}-s_{m_{1} i}\right)\left(s_{m_{0} i}-t_{m_{1} i}\right) \geq 0, \quad m_{0} \neq m_{1}, \quad r_{m_{0} i}=r_{m_{1} i}, \quad m=1,2, \cdots, M \\
\left(t_{m_{0} i}-s_{m_{1} i}\right)\left(t_{m_{0} i}-t_{m_{1} i}\right) \geq 0, \quad m_{0} \neq m_{1}, \quad r_{m_{0} i}=r_{m_{1} i}, \quad m=1,2, \cdots, M \\
D=\sum_{n} \sum_{m} \sum_{i}\left(s_{m(i+1)}-t_{m i}\right), \quad m=1,2, \cdots, M_{n}, \quad i=1, \cdots, k_{m}-1
\end{gathered}
$$

Eq. (1) is the objective function, aims to minimize the makespan. Eq. (2) is the constrain of continuous casting. Eq. (3) is the constrain of the process time. Eq. (4) means the constrain of the beginning time and the finish time when all the processes are processed on time. Eqs. (5) and (6) are the time constraints for the buffer processes. Eqs. (8) and (9) mean that it only allows one task for the same time in the same process. Eq. (9) limit the waiting time of the cast. Eq. (9) could be removed if there is no waiting time according to the result of Eq. (4).

\section{HYBRID ALGORITHM}

In recent years, some researches focused on the optimization of the SCC process scheduling problems based on Particle Swarm Optimization (PSO) algorithm. However, the standard 
PSO algorithm is greatly affected by the initial group of particle swarm, and it needs to expand population size and increase the number of iterations to ensure ergodicity of the algorithm in dealing with large-scale problem. To solve the problem, this paper studies the quantum particle swarm optimization (QPSO) algorithm to solve SSC production scheduling problem and put forward improved algorithm strategies.

\subsection{Quantum-behaved particle swarm optimization}

PSO is a new evolutionary algorithm which has been developed in recent years. Kennedy and Eberhart were inspired from the behaviour of bird populations from predation, and then proposed PSO [20]. In PSO, the solution for each optimization problem is a particle. Each particle has an adaptive value and a speed of its fighting. The positions of particles are identified randomly and the velocity is initialized as 0 in the first iteration. At first the particles update their speed and new position with the formula below:

$$
\begin{gathered}
V_{i, j}(t+1)=w \cdot V_{i, j}(t)+c_{1} \cdot r_{1, i}(t) \cdot\left[P_{i, j}(t)-X_{i, j}(t)\right]+c_{2} \cdot r_{2, i}(t) \cdot\left[P_{g, j}(t)-X_{i, j}(t)\right] \\
X_{i, j}(t+1)=X_{i, j}(t)+V_{i, j}(t+1)
\end{gathered}
$$

Literature on the convergence of the PSO shown that in order to guarantee the convergence of the algorithm, each particle should convergence particle attractor. That is [21]:

$$
\begin{gathered}
C_{i, j}(t)=\left(c_{1} \cdot r_{1} \cdot P_{i, j}(t)+c_{2} \cdot r_{2} \cdot P_{g, j}(t)\right) /\left(c_{1} \cdot r_{1}+c_{2} \cdot r_{2}\right), \quad j=1,2, \cdots, d, c_{1}, c_{2} \in U(0,1) \\
C_{i, j}(t)=\alpha \cdot P_{i, j}(t)+(1-\alpha) \cdot P_{g, j}(t), \alpha \in U(0,1)
\end{gathered}
$$

$P_{i}$ is the personal best particle and $P_{g}$ is the global best particle, where $\alpha=\left(c_{1} \cdot r_{1}\right) /\left(c_{1} \cdot r_{1}+c_{2} \cdot r_{2}\right)$. It can be seen that the local attractor is a stochastic attractor of particle $i$ that lies in a hyper-rectangle with $P_{i}$ and $P_{g}$ being two ends of its diagonal.

On the basis of the study of the convergence process of particles, Sun et al. put forward a quantum-behaved particle swarm optimization algorithm (QPSO) [22]. Because of the removal of the velocity vector, the QPSO algorithm has fewer parameters, and optimizes the search strategy.

According to the literature [23], the quantum state of the quantum state is described by a wave function $\psi(\vec{x}, t)$. Then a global point is introduced in QPSO [24]. Global point mbest is identified as the mean of the Pbest positions of all particles. And the formulation is shown in Eq. (14):

$$
\text { mbest }(t)=\left(\text { mbest }_{1}(t), \text { mbest }_{2}(t), \ldots \text { mbest }_{d}(t)\right)=\left(\frac{1}{n} \sum_{i=1}^{n} P_{i, 1}(t), \frac{1}{n} \sum_{i=1}^{n} P_{i, 2}(t), \ldots, \frac{1}{n} \sum_{i=1}^{n} P_{i, d}(t)\right)
$$

Then, the new position of a particle is:

$$
X_{i, j}(t+1)=C_{i, j}(t) \pm \beta \cdot \mid \text { mbest }_{j}-X_{i, j}(t) \mid \cdot \ln (1 / u), u=\operatorname{rand}(0,1)
$$

where $\beta$ is the contraction-expansion coefficient.

Generally speaking, the QPSO is structured in three steps: firstly, the positions of the particles are initialized randomly, and $P_{i}$ of an initial position of particles is initialized as 0 . Secondly, the objective functions, $P_{i}$ and $P_{g}$ are identified. Thirdly, the new position and velocity are given.

\subsection{Decoding method}

The first step for the QPSO algorithm to solve the SCC process is the decoding. For the general SCC process model, the array decoding method can be used. The number of charge can be denoted by the number of array, and the number of cast can be stored in the array. As shown in Fig. 2. 


\begin{tabular}{cccc|c|c|c|c|c|} 
Charge & 1 & 2 & $\ldots$ & $n$ & $\ldots$ & \multicolumn{1}{c}{$N$} \\
Cast & 1 & 3 & $\ldots$ & $k$ & $\ldots$ & 2 & 1 \\
\hline
\end{tabular}

Figure 2: Array decoding example.

According to the characteristics of the SCC process model in this paper, the array decoding method couldn't cover all processes. So a matrix decoding method is proposed, as shown in Fig. 3.

\begin{tabular}{|c|c|c|c|c|c|c|c|c|c|}
\hline Charge & 1 & 2 & 3 & 4 & $n$ & \multicolumn{4}{|c|}{$N-1 N$} \\
\hline LD & 2 & 1 & 1 & 2 & $\ldots$ & 3 & 1 & 3 & 2 \\
\hline AR & 0 & 2 & 0 & 1 & $\ldots$ & 2 & 0 & 0 & 0 \\
\hline LF & 2 & 0 & 0 & 0 & $\ldots$ & 0 & 1 & 1 & 0 \\
\hline RH & 0 & 0 & 1 & 0 & $\ldots$ & 0 & 2 & 1 & 2 \\
\hline $\mathrm{CW}$ & 1 & 2 & 1 & 1 & $\ldots$ & 2 & 1 & 2 & 2 \\
\hline $\mathrm{CC}$ & 2 & 3 & 2 & 3 & $\ldots$ & 1 & 3 & 1 & 3 \\
\hline
\end{tabular}

Figure 3: Matrix decoding example.

There is a $6 \times n$ matrix in Fig. 3. And a row of the matrix expresses a process and a column of the matrix express all processes of a charge. While, the first row means LD process, the second row means AR process, the third row means LF process, the fourth row means $\mathrm{RH}$ process, the fifth row means CW process, and the last row means $\mathrm{CC}$ process. And, the first column means the process routing of charge 1 is: LD2-LF2-CW1-CC2, the second column means the process routing of charge 2 is: LD1-AR2-CW2-CC3, the third column means the process routing of charge 3 is: LD1-RH1-CW1-CC2, and the $N-1$ column means the process routing of charge $N-1$ is: LD3-LF1-RH1-CW2-CC1. In addition, adjust the machine number of each process can get more production path.

\section{COMPUTATIONAL EXPERIMENTS}

\subsection{Parameter selection}

The popsize, inertia weight parameters, as well as the parameters $c_{1}$ and $c_{2}$ need to be determined according to the literature [25]. In addition, there is a new parameter contraction-expansion coefficient $\beta$ in QPSO algorithm. Four functions which are widely used to testify the performance of the algorithm are employed to test the QPSO algorithm under different parameter combinations of properties. This article set the popsize to 50 . The function expressions are shown in Table II.

Parameter design strategy is as follows:

a) EX1: $c_{1}=c_{2}=2, \omega=[0.4,0.9], \beta=0.5$;

b) EX2: $c_{1}=1.85, c_{2}=2, \omega=[0.4,0.9], \beta=1$;

c) EX3: $c_{1}=c_{2}=2, \omega=[0.38,0.99], \beta=0.5$;

d) EX4: $c_{1}=1.85, c_{2}=2, \omega=[0.38,0.99], \beta=1$.

50 independent simulation experiments are made for four kinds of parameter strategies respectively, and it stops when the target error arrives. 
Table II: Function expressions.

\begin{tabular}{|c|c|c|c|c|c|}
\hline Function & Function expression & Dimension & Value area & $\begin{array}{c}\text { Theoretical } \\
\text { extreme value }\end{array}$ & $\begin{array}{c}\text { Target } \\
\text { error }\end{array}$ \\
\hline Sphere & $f(x)=\sum_{i=1}^{n} x_{i}^{2}$ & $\mathbf{3 0}$ & {$[-100,100]^{n}$} & $\mathbf{0}$ & $\mathbf{1 0}^{-2}$ \\
\hline Rastrigin & $f(x)=\sum_{i=1}^{n}\left(x_{i}^{2}-10 \cos \left(2 \pi x_{i}\right)+10\right)$ & $\mathbf{3 0}$ & {$[-5.12,5.12]^{n}$} & $\mathbf{0}$ & $\mathbf{1 0}^{-2}$ \\
\hline Griewank & $f(x)=\frac{1}{4000} \sum_{i=1}^{n} x_{i}^{2}-\prod_{i=1}^{n} \cos \left(\frac{x_{i}}{\sqrt{i}}\right)+1$ & $\mathbf{3 0}$ & {$[-600,600]^{n}$} & $\mathbf{0}$ & $\mathbf{1 0}^{-2}$ \\
\hline Rosenbrock & $f(x)=\sum_{i=1}^{n-1}\left(100\left(x_{i+1}+x_{i}^{2}\right)+\left(x_{i}-1\right)^{2}\right)$ & $\mathbf{3 0}$ & {$[-30,30]^{n}$} & $\mathbf{0}$ & $\mathbf{1 0}^{-2}$ \\
\hline
\end{tabular}

The success rate of convergence $(S u c)$, the minimum number of iterations (Min), the largest number of iterations (Max) and the average number of iterations (Mean), and calculate the standard deviation $(S t . D)$ are recorded and the sequence is given according to the $S t . D$. The parameter selection experiment results are shown in Table III.

Table III: Parameter selection experiment result.

\begin{tabular}{|c|c|c|c|c|c|c|c|}
\hline Function & Type & Suc (\%) & Min & Max & Mean & St. D & Sequence \\
\hline \multirow{4}{*}{ Sphere } & EX1 & 100 & 582 & 2440 & 1394 & 277.4 & 4 \\
\cline { 2 - 8 } & EX2 & 100 & 321 & 583 & 414 & 81 & 2 \\
\cline { 2 - 8 } & EX3 & 100 & 201 & 374 & 264 & 51.4 & 1 \\
\cline { 2 - 8 } & EX4 & 99.7 & 424 & 992 & 718 & 178.2 & 3 \\
\hline \multirow{5}{*}{ Rastrigin } & EX1 & 100 & 990 & 2776 & 1912 & 365 & 4 \\
\cline { 2 - 8 } & EX2 & 100 & 142 & 649 & 401 & 119.3 & 2 \\
\cline { 2 - 8 } & EX3 & 99.8 & 141 & 571 & 342 & 78.5 & 1 \\
\cline { 2 - 8 } & EX4 & 100 & 322 & 1720 & 1036 & 127 & 3 \\
\hline & EX1 & 100 & 152 & 787 & 372 & 27.8 & 2 \\
\cline { 2 - 8 } & EX2 & 100 & 53 & 153 & 119 & 29 & 4 \\
\cline { 2 - 8 } & EX3 & 100 & 34 & 112 & 67 & 25 & 1 \\
\cline { 2 - 8 } & EX4 & 100 & 102 & 216 & 130 & 28.6 & 3 \\
\hline \multirow{5}{*}{ Rosenbrock } & EX1 & 100 & 568 & 1306 & 898 & 139 & 1 \\
\cline { 2 - 8 } & EX2 & 100 & 250 & 1456 & 873 & 333 & 3 \\
\cline { 2 - 8 } & EX3 & 100 & 247 & 871 & 672 & 184.5 & 2 \\
\cline { 2 - 8 } & EX4 & 100 & 753 & 2166 & 1573 & 431 & 4 \\
\hline
\end{tabular}

Table III shows that the performance of EX3 is the best among all parameter selection experiments, so the parameters of EX3 $\left(c_{1}=c_{2}=2, \omega=[0.38,0.99], \beta=0.5\right)$ are selected for the further experiment. 


\subsection{Simulation experiment}

The experimental data come from the actual production situation of a large-size iron \& steel company in China. Select the order and production data in the second quarter of 2015 (April, May and June) of the factory for experiments. Experiments are carried out in a parallel state for ten times, and calculate the average, as shown in Table IV.

Table IV: Calculation results.

\begin{tabular}{|l|c|r|r|}
\hline Sample & $\begin{array}{c}\text { Calculate times } \\
(\mathrm{min})\end{array}$ & $\begin{array}{c}\text { PSO } \\
\left(10^{3} \mathrm{~h}\right)\end{array}$ & $\begin{array}{r}\text { QPSO } \\
\left(10^{3} \mathrm{~h}\right)\end{array}$ \\
\hline April & 5 & 7.99 & 6.89 \\
\hline May & 5 & 6.85 & 5.78 \\
\hline June & 5 & 8.03 & 6.54 \\
\hline Second quarter & 10 & 25.41 & 20.15 \\
\hline
\end{tabular}

From the data of Table IV, compared with the traditional PSO algorithm, the QPSO that proposed in this paper can get better results. Therefore, the QPSO algorithm has better performance in the process of dealing with large-scale SCC process problem.

\section{CONCLUSIONS}

Optimization of the scheduling SCC processes can reduce the energy consumption and the productions cost, as well as increase the profit, so many iron \& steel companies have studied the SCC production process for continuous improvement. We study the optimal models for the SCC problem. It is very difficult to get a good performance solution in practice in iron \& steel production, especially for the large-scale problems. We employ QPSO algorithm and put forward the improved algorithm strategies to solve SSC production scheduling problem. It has been proved that comparing with the PSO algorithm, the QPSO algorithm is a very efficient approach and it can help the companies get the scheduling results in the shorter total computing time and better performances for the SCC process scheduling problem, especially when dealing with large scale problem. In addition, the QPSO algorithm can escape effectively from local optimum compared with PSO algorithm. The simulation results also confirm the conclusions.

\section{ACKNOWLEDGEMENT}

The work has been funded by the Fundamental Research Funds for Natural Science Foundation of Zhejiang Province (LQ15G030002) and Research Funds for Ministry of Education of the Republic of China (15YJA630044).

\section{REFERENCES}

[1] Tang, L.-X.; Luh, P. B.; Liu, J.-Y.; Fang, L. (2002). Steel-making process scheduling using Lagrangian relaxation, International Journal of Production Research, Vol. 40, No. 1, 55-70, doi: $10.1080 / 00207540110073000$

[2] Sun, L.-L.; Wang, X.-Y. (2013). Application of mix optimization scheduling approach for steelmaking-continuous casting process based on actual steelmaking industry, Journal of Iron and Steel Research, International, Vol. 20, No. 10, 1-9, doi:10.1016/s1006-706x(13)60168-5

[3] Harjunkoski, I.; Grossmann, I. E. (2001). A decomposition approach for the scheduling of a steel plant production, Computers \& Chemical Engineering, Vol. 25, No. 11-12, 1647-1660, doi: $\underline{10.1016 / \mathrm{s} 0098-1354(01) 00729-3}$ 
Yang, Qiu, Luo, Shen: Simulating Schedule Optimization Problem in Steelmaking ...

[4] Bellabdaoui, A.; Teghem, J. (2006). A mixed-integer linear programming model for the continuous casting planning, International Journal of Production Economics, Vol. 104, No. 2, 260-270, doi:10.1016/j.ijpe.2004.10.016

[5] Missbauer, H.; Hauber, W.; Stadler, W. (2009). A scheduling system for the steelmaking-continuous casting process. A case study from the steel-making industry, International Journal of Production Research, Vol. 47, No. 15, 4147-4172, doi:10.1080/ $\underline{00207540801950136}$

[6] Pacciarelli, D.; Pranzo, M. (2004). Production scheduling in a steelmaking-continuous casting plant, Computers \& Chemical Engineering, Vol. 28, No. 12, 2823-2835, doi:10.1016/ j.compchemeng.2004.08.031

[7] Atighehchian, A.; Bijari, M.; Tarkesh, H. (2009). A. novel hybrid algorithm for scheduling steel-making continuous casting production, Computers \& Operations Research, Vol. 36, No. 8, 2450-2461, doi:10.1016/j.cor.2008.10.010

[8] Tang, L.-X.; Liu, J.-Y.; Rong, A.-Y.; Yang, Z.-H. (2001). A review of planning and scheduling systems and methods for integrated steel production, European Journal of Operational Research, Vol. 133, No. 1, 1-20, doi:10.1016/s0377-2217(00)00240-x

[9] Dutta, G.; Fourer, R. (2001). A survey of mathematical programming applications in integrated steel plants, Manufacturing \& Service Operations Management, Vol. 3, No. 4, 387-400, doi: $10.1287 / \mathrm{msom} \cdot 3.4 .387 .9972$

[10] Lee, H. S.; Murthy, S. S.; Haider, S. W.; Morse, D. V. (1996). Primary production scheduling at steelmaking industries, IBM Journal of Research and Development, Vol. 40, No. 2, 231-252

[11] Li, J.; Xiao, X.; Tang, Q.-H.; Floudas, C. A. (2012). Production scheduling of a large-scale steelmaking continuous casting process via unit-specific event-based continuous-time models: Short-term and medium-term scheduling, Industrial \& Engineering Chemistry Research, Vol. 51, No. 21, 7300-7319, doi:10.1021/ie2015944

[12] Lin, X.-X.; Floudas, C. A.; Modi, S.; Juhasz, N. M. (2002). Continuous-time optimization approach for medium-range production scheduling of a multiproduct batch plant, Industrial \& Engineering Chemistry Research, Vol. 41, No. 16, 3884-3906, doi:10.1021/ie011002a

[13] Janak, S. L.; Floudas, C. A.; Kallrath, J.; Vormbrock, N. (2006). Production scheduling of a large-scale industrial batch plant. I. Short-term and medium-term scheduling, Industrial \& Engineering Chemistry Research, Vol. 45, No. 25, 8234-8252, doi:10.1021/ie0600588

[14] Janak, S. L.; Floudas, C. A.; Kallrath, J.; Vormbrock, N. (2006). Production scheduling of a large-scale industrial batch plant. II. Reactive scheduling, Industrial \& Engineering Chemistry Research, Vol. 45, No. 25, 8253-8269, doi:10.1021/ie0600590

[15] Chen, H.-X.; Chu, C.-B.; Proth, J.-M. (1998). An improvement of the Lagrange relaxation approach for job shop scheduling: a dynamic programming method, IEEE Transactions on Robotics and Automation, Vol. 14, No. 5, 786-95, doi:10.1109/70.720354

[16] Fisher, M. L. (1981). The Lagrange relaxation method for solving integer programming problems, Management Science, Vol. 27, No. 1, 1-18, doi:10.1287/mnsc.27.1.1

[17] Tang, L.-X.; Liu, J.-Y.; Rong, A.-Y.; Yang, Z.-H. (2000). A mathematical programming model for scheduling steelmaking-continuous casting production, European Journal of Operational Research, Vol. 120, No. 2, 423-435, doi:10.1016/s0377-2217(99)00041-7

[18] Luh, P. B.; Wang, J. H.; Wang, J. L.; Tomastik, R. N.; Howes, T. D. (1997). Near-optimal scheduling of manufacturing systems with presence of batch machines and setup requirements, CIRP Annals - Manufacturing Technology, Vol. 26, No. 1, 397-402, doi:10.1016/s00078506(07)60851-8

[19] Hoitomt, D. J.; Luh, P. B.; Pattipati, K. R. (1993). A practical approach to job shop scheduling problems, IEEE Transactions on Robotics and Automation, Vol. 9, No. 1, 1-13, doi:10.1109/ 70.210791

[20] Kennedy, J.; Eberhart, R. C. (2001). Swarm Intelligence, Academic Press - Morgan Kaufman Publishers, San Francisco

[21] Liu, J.; Sun, J.; Xu, W.-B. (2006). Quantum-behaved particle swarm optimization with adaptive mutation operator, Jiao, L.-C.; Wang, L.-P.; Gao, X.-B; Liu, J.; Wu, F. (Eds.). Advances in Natural Computation, $2^{\text {nd }}$ International Conference ICNC 2006 Proceedings, Part I, Vol. 4221 (Lecture Notes in Computer Science), 959-967, doi:10.1007/11881070_126

[22] Jin, X.; Liang, Y.-Q.; Tian, D.-P.; Zhuang, F.-Z. (2013). Particle swarm optimization using dimension selection methods, Applied Mathematics and Computation, Vol. 219, No. 10, 5185-5197, doi:10.1016/j.amc.2012.11.020 
[23] Tang, D.-Y.; Cai, Y.-M.; Zhao, J.; Xue, Y. (2014). A quantum-behaved particle swarm optimization with memetic algorithm and memory for continuous non-linear large scale problems, Information Sciences, Vol. 289, 162-189, doi:10.1016/j.ins.2014.08.030

[24] Sun, J.; Xu, W.-B.; Feng, B. (2004). A global search strategy of quantum-behaved particle swarm optimization, IEEE Conference on Cybernetics and Intelligent Systems, Vol. 1, 111-116, doi:10.1109/iccis.2004.1460396

[25] Zhang, W.-X.; Li, T.-K. (2010). Integrated batch planning optimization based on particle swarm optimization and constraint satisfaction for steel production, Computer Integrated Manufacturing Systems, Vol. 16, No. 4, 840-846, doi:10.13196/j.cims.2010.04.170.zhangwx.002 